REVIEW

\title{
Role of surfactant protein A in non-infectious lung diseases
}

\author{
Hisatsugu Goto, Atsushi Mitsuhashi, and Yasuhiko Nishioka \\ Department of Respiratory Medicine and Rheumatology, Institute of Health Biosciences, the University \\ of Tokushima Graduate School, Tokushima, Japan
}

\begin{abstract}
Surfactant protein A (SP-A) is a large multimeric protein found in the airways and alveoli of the lungs. SP-A is a member of the collectin family of proteins, characterized by $\mathrm{NH}_{2}$-terminal collagen-like regions and $\mathrm{COOH}$-terminal lectin domains. Although other surfactant proteins such as SP-B function to reduce surface tension in the lungs, SP-A as well as SP-D regulates the pulmonary immune response. To date, a number of studies have shown the immunoregulatory function of SP-A, mainly in the field of infectious diseases. By binding to a wide variety of pathogens, SP-A opsonizes and enhances pathogen uptake by phagocytes. In addition to the effect on pathogens, recent studies have shown that SP-A also modulates lung immune system in the area of non-infectious lung diseases. In this review, the potential role of SP-A in the multiple aspects of pulmonary host defense will be discussed, focusing mainly on non-infectious lung diseases such as acute and chronic pulmonary fibrosis and lung cancer. J. Med. Invest. 61 : 1-6, February, 2014
\end{abstract}

Keywords : surfactant protein A, non-infectious lung injury, lung cancer

\section{INTRODUCTION}

Pulmonary surfactant was initially identified as a lipoprotein complex that reduces surface tension at the air-liquid interface of the lung $(1,2)$. This definition has been reassessed with recent studies showing that surfactant also functions in pulmonary host defense. Surfactant is mostly composed of phospholipids that are essential for reducing surface tension at the air-liquid interface of the lung. About 10\% of surfactant consists of protein ; four surfactant proteins (SPs) have been defined: SP-A, SP-B, SP-C and SP-D. Among these proteins, SP-B and SP-C are small and hydrophobic, and SP-B is essential for the ability of surfactant to reduce surface tension (3). On the other hand, the host-defence functions

Received for publication November 29, 2013 ; accepted December 25, 2013.

Address correspondence and reprint requests to Hisatsugu Goto, Department of Respiratory Medicine and Rheumatology, Institute of Health Biosciences, the University of Tokushima Graduate School, 3-18-15 Kuramoto-cho, Tokushima 770-8503, Japan and Fax : +81-88-633-2134. of surfactant are primarily mediated by SP-A and SP-D, which are members of the collectin family of proteins.

Collectins are distinguished by their amino (N)terminal collagen-like regions that have a repeating triple helix of Gly-X-Y triplets, where $\mathrm{X}$ denotes any amino acid and $\mathrm{Y}$ is often a hydroxyproline residue. The carboxy-terminal domains of the collectins all have $\mathrm{C}$-type (calcium-dependent) lectin activity. The lectin domains mediate the interaction of collectins with a wide variety of pathogens. The most well-understood consequence of this interaction is pathogen opsonization and enhanced uptake by phagocytes. SP-A and SP-D are synthesized as primary translation products of approximately 26-36 $\mathrm{kDa}$ and $43 \mathrm{kDa}$, respectively. The collagen-like domain is N-terminal to a coiled-coil structure that precedes the lectin domain. The collectins are assembled as trimeric subunits, which multimerize to varying degrees. SP-A is mainly an octadecamer and forms a bouquet-like structure, whereas SP-D forms a dodecamer (Figure 1). SP-A structurally 
A.

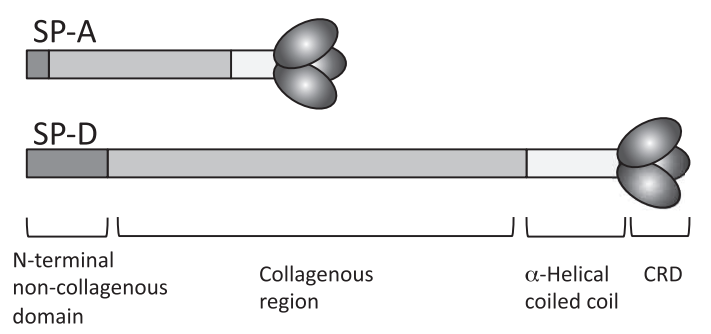

B.

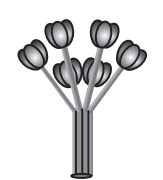

SP-A

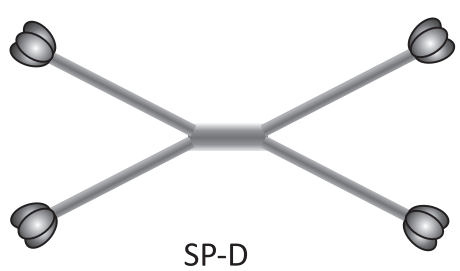

SP-D
Figure 1. SP-A and SP-D are members of a family of proteins known as collectins. (A) Collectins have collagen-like amino (N) terminal regions and C-type (calcium dependent) carbohydraterecognition domains (CRDs). Collectins consist of structural subunits that are composed of trimeric polypeptide chains. The trimers are assembled into oligomers. SP-A is octadecamer (18mer), consisting of six trimeric subunits. SP-D is a dodecamer (12-mer), consisting of four trimeric subunits. The models are not drawn to scale.

resemble the first component of complement, $\mathrm{C} 1 \mathrm{q}$, however, $\mathrm{C} 1 \mathrm{q}$ does not contain a lectin domain, although it does have a collagen-like N-terminal triple helix. In humans, the genes that encode SP-A and SP-D have been mapped to a cluster on the long arm of chromosome 10 (4-7).

Since SP-A and SP-D are the members of the collectin family, the immunoregulatory functions of SP-A and SP-D have been studied mainly in the field of infectious diseases. They are able to bind to a variety of bacteria, viruses, allergens and apoptotic cells and thereby function as opsonins to enhance the uptake of these cells and particles. Some pathogens are aggregated by SP-A and/or SP-D. They are also reported to have direct effects on immune cells and modulate the production of cytokines and inflammatory mediators $(8,9)$. On the other hand, recent clinical and experimental studies suggest that they are also involved in non-infectious lung diseases such as pulmonary fibrosis and lung cancer. In this review, we focus and discuss on diverse functions of SP-A in various lung diseases. An emphasis is placed on recent studies showing that SP-A has novel functions in regulating epithelial cell apoptosis in mouse bleomycin-induced acute lung injury mod$\mathrm{el}$, and controlling the polarization of macrophages in lung cancer model.

\section{SP-A AND ACUTE NON-INFECTIOUS LUNG INJURY}

Acute respiratory distress syndrome (ARDS) is a clinical syndrome with acute lung injury characterized by significant hypoxemia with bilateral pulmonary infiltrates consistent with edema $(10,11)$. ARDS is caused by multiple etiologies including non-infectious lung diseases such as acute interstitial pneumonia and chemical pneumonia, as well as infectious diseases such as microbial pneumonia and sepsis. Despite decades of intense investigation, the fundamental mechanisms that initiate and control ARDS have not been elucidated, and conventional treatment plans such as high-dose glucocorticoid therapy is not effective in many cases and overall 28-day mortality of ARDS is fairly high (25-40\%).

Several clinical studies reported the changes of SP-A expression in ARDS patients. They have demonstrated that SP-A concentration in the bronchoalveolar lavage (BAL) fluid was significantly lower in both established ARDS patients and patients at-risk for ARDS, compared to healthy volunteers $(12,13)$. On the other hand, SP-A levels were increased in the serum of patients with ARDS (12). These findings suggested that SP-A might play an important role in the pathogenesis of non-infectious lung injury. We therefore evaluated the contribution of SP$A$ in the pathogenesis of non-infectious lung injury using mouse bleomycin-induced lung injury model. We compared the development of bleomycin-induced acute lung injury in wild-type (WT) and SP-A -/- mice, and found that: (a) SP-A -/- mice were more susceptible to bleomycin induced death; (b) SP-A -/- mice exhibited higher level of inflammatory cytokine and high mobility group box (HMGB) 1 expression as well as increased vascular permeability compared to WT mice ; and (c) exogenous SP-A administration rescued the phenotype of SP-A -/- mice. In combination with in vitro experiments, we have also shown that SP-A reduces apoptosis induced by bleomycin (14). These results suggested the importance of SP-A in the pathogenesis of noninfectious acute lung injury. Although the precise mechanism by which SP-A regulates epithelial cell apoptosis (especially the early stage of apoptosis ; see ref. 14) needs to be further determined, this observation might also explain the increased vascular permeability and HMGB1 levels in SP-A -/- mice compared to WT mice subject to bleomycin treatment. As maintaining the integrity of the airway epithelium is a crucial step in the regulation of acute 
lung injury, SP-A might have the defensive role in the pathogenesis of ARDS by regulating the epithelial cell apoptosis to maintain the integrity of the alveolar epithelium.

\section{SP-A AND LUNG CANCER}

Lung cancer is the major cause of malignancyrelated death worldwide. The mortality rate is 80 $90 \%$, which makes this disease the leading cause of cancer-related death (15). The high mortality of this disease is primarily due to the difficulty of early diagnosis, the high metastatic potential, and poor responses to chemical or radiation therapy. Since there is no established curative therapy for advanced lung cancer to date, clinical management is palliative in many cases. Therefore, it is crucial to investigate and understand the underlying biological and molecular mechanisms of lung cancer progression.

In clinical studies, SP-A was expressed in approximately $49 \%$ of primary non-small cell lung carcinoma (16) and is used as specific marker of carcinoma that originates in type II pneumocytes. Additionally, a previous study demonstrated that deletion of the $S P$-A gene in non-small lung cancer cells was associated with tumor progression (17). Tsutsumida $e t$ $a l$. found that lung adenocarcinoma patients with relatively high MUC1 mucin expression and low SPA expression in cancer cells had poor outcome (18). These studies demonstrate that, in addition to use as a diagnostic marker, SP-A expression in lung cancer cells could be the useful biomarker of good prognosis. However, the role of SP-A in lung cancer has not been extensively studied and the mechanisms by which SP-A controls lung cancer progression is still unknown.

To determine the role of SP-A in lung cancer pathogenesis, we have generated SP-A over-expressing human lung adenocarcinoma cells, and showed that: (a) SP-A expression in cancer cells suppressed progression of lung adenocarcinoma in both xenograft and lung metastasis models ; (b) SPA inhibited lung cancer progression not by its direct effect on tumor cells but by regulating host microenvironment, including macrophages and natural killer (NK) cells ; (c) SP-A increased the number of M1 tumor-associated macrophages (TAMs) in the tumor microenvironment, resulting in NK cell recruitment and activation within tumor tissue (Figure 2). These results suggested new immunoregulatory functions of SP-A, which is frequently expressed in

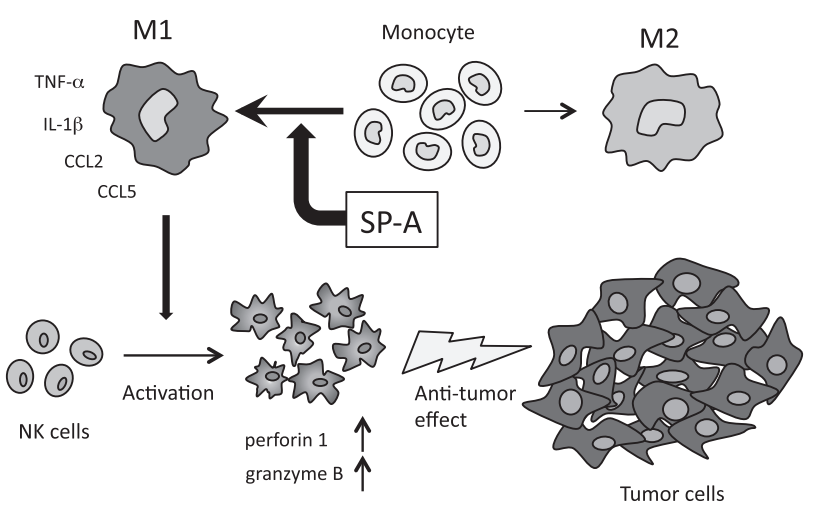

Figure 2. Possible role of SP-A on tumor progression. Although the precise mechanism by which SP-A regulates the polarization of TAMs is still unknown, SP-A increases the number of M1 TAMs in the tumor microenvironment, resulting in NK cell recruitment and activation within tumor tissue. Activated NK cells produce perforin 1 and granzyme B, which in turn can inhibit tumor growth. SP-A was confirmed not to have direct effect on NK cells (ref. 19).

pulmonary adenocarcinoma (19).

Considering the character of TAMs, it is now generally accepted that TAMs usually polarize to M2 and represent pro-tumoral functions (20). Indeed, we have seen in our study that approximately $60 \%$ of TAMs had M2 phenotype in vector-transduced tumors. However, when tumor cells expressed SP-A, this M1/M2 balance was reversed, and M1 anti-tumor (pro-inflammatory) macrophages became dominant. Subsequent analyses indicated that SP-A aided in making the TAMs M1-dominant by increasing the number of recruited M1 macrophages rather than shifting the M2 TAMs into M1 phenotype in the tumor microenvironment. In the field of infection, it is well known that the macrophages are one of the target cells that SP-A interacts with to regulate infectious inflammation. From this point of view, there might be a common pathways for SP-A to regulate macrophage-related inflammation between infectious lung diseases and lung cancer. For instance, SP-A may enhance the binding of cytokines to their respective receptors. SP-A is reported to bind to several receptors including Toll-like receptor (TLR) 2 and 4 , and regulate inflammatory responses induced by pathogen-derived products such as peptidoglycan and LPS via TLRs (21-23). In addition to its role in TLR-mediated cellular responses induced by infectious challenges, it is very possible that SP-A regulates the function of TAMs in the tumor microenvironment through the interaction with TLRs. However, it is also considerable that the story is not so simple. We also showed in this study that SP-A activated only circulating monocytes/macrophages 
while it showed no effect on resident alveolar macrophages (AMs) in cytokine expression. In the lung, the resident AMs are thought to acquire the tolerance against SP-A as they are continuously contacted by SP-A, which could be a plausible explanation, as the host needs to be protected from the overzealous inflammation in the lungs. Further studies are needed to understand the precise molecular mechanisms of the diverse and cell-specific function of SP-A against macrophages in the context of SP-A and lung cancer.

\section{GENETIC VARIATIONS IN SP-A}

Two functional genes of SP-A were detected in previous report (24) ; SFTPA1 and SFTPA2 that encode SP-A1 and SP-A2 protein, respectively. Although human SP-A1 and SP-A2 have a 96\% degree of similarity at the protein level, these genes were differentially regulated by development (25) and have a minor difference in carbohydrate-binding activity (26), resulting in the functional differences. For example, SP-A2 exhibits a higher level of activity than SP-A1 in its ability to enhance inflammatory gene induction and pathogen clearance, potentially due to the increased stability of the protein $(27,28)$. Although a comprehensive documentation of the allelic variations of all SPs is beyond the scope of this review, we will introduce several common polymorphisms that occur in SP-A, as we are in the period to begin to understand the molecular mechanisms by which these polymorphisms affect SP function.

Selman et al. has reported that one SP-A1 $\left(6 \mathrm{~A}^{4}\right)$ allele and the single-nucleotide polymorphisms (SNPs) that characterize the $6 \mathrm{~A}^{4}$ allele were found with higher frequency in idiopathic pulmonary fibrosis (29). More recently, Maitra et al. reported that BAL fluid from humans heterozygous for a missense mutation in SFTPA2 which changes glycine at position 231 to valine (G231V) contained more transforming growth factor (TGF)- $\beta 1$ than control samples, and expression of mutant SP-A2 in lung epithelial cells led to secretion of latent TGF$\beta 1$, which was capable of autocrine and paracrine signaling (30). These data suggest that therapeutic targeted to block the pathway induced by mutant SP-A2 might be especially beneficial for the molecularly defined subgroup of patients with pulmonary fibrosis.

\section{CONCLUDING REMARKS}

From decades ago, surfactant was recognized as a soap-like substance that reduced surface tension in the lung and made breathing easier. With the development of molecular and biological techniques, it was discovered that SP-A was structurally homologous to an immune protein of the complement cascade, C1q. Since then, an entire family of proteins has been identified, and the role of the innate immune system has garnered increasing attention. After the generation of knock-out mice, in vivo and in vitro data accumulation was accelerated, and now it is well known that SP-A (and SP-D) has the defensive role in infectious lung diseases by mediating various immune-cell functions. More recently, studies have shown novel roles for these proteins in noninfectious lung diseases. As presented in this review, SP-A plays a role in regulating apoptosis in the model of acute lung injury and controlling the polarization of macrophages in lung cancer model. Modern high molecular technologies would make us possible to explore more deeply in the genetic variances in SPs in various diseases, which also has a potential to accelerate the understanding of SP function. Although there are still many obstacles, the studies described in this review support the intriguing possibility that therapeutic strategy targeting SP-A might be efficacious for the treatment of non-infectious lung diseases as well as infectious diseases.

\section{CONFLICT OF INTEREST}

The authors have declared no conflicts of interest.

\section{ACKNOWLEDGEMENTS}

The results of our studies presented in this review were done in collaboration with the late Dr. Jo Rae Wright (Duke University, NC). We greatly appreciate and honor her contribution to the research of surfactant molecular biology.

\section{REFERENCES}

1. Pattle RE : Properties, function and origin of the lining layer. Nature $175:$ 1125-1126, 1955

2. Clements JA : Surface tension of lung extracts. Proc Soc Exp Biol Med 95 : 170-172, 1957 
3. Nogee LM : Alterations in SP-B and SP-C expression in neonatal lung disease. Annu Rev Physiol 66 : 601-623, 2004

4. Holmskov U, Thiel S, Jensenius JC : Collectins and ficolins : humoral lectins of the innate immune defense. Annu Rev Immunol 21 : 547578, 2003

5. Bruns G, Stroh H, Veldman GM, Latt SA, Floros J : The $35 \mathrm{kD}$ pulmonary surfactant-associated protein is encoded on chromosome 10 . Hum Genet 76 : 58-62, 1987

6. Fisher JH, Kao FT, Jones C, White RT, Benson $\mathrm{BJ}$, Mason RJ : The coding sequence for the 32,000-dalton pulmonary surfactant-associated protein $\mathrm{A}$ is located on chromosome 10 and identifies two separate restriction-fragmentlength polymorphisms. Am J Hum Genet 40 : 503-511, 1987

7. Crouch E, Rust K: Veile R, Donis-Keller H, Grosso L: Genomic organization of human surfactant protein D (SP-D). J Biol Chem 268 : 2976-2983, 1993

8. Wright JR: Immunoregulatory functions of surfactant proteins. Nat Rev Immunol 5 : 58-68, 2005

9. Pastva AM, Wright JR, Williams KL : Immunomodulatory roles of surfactant proteins A and $\mathrm{D}$ : implications in lung disease. Proc Am Thorac Soc $4: 252-257,2007$

10. Ashbaugh DG, Bigelow DB, Petty TL, Levine $\mathrm{BE}$ : Acute respiratory distress in adults. Lancet $2: 319-323,1967$

11. Wheeler AP, Bernard GR : Acute lung injury and the acute respiratory distress syndrome : a clinical review. Lancet 369 : 1553-1564, 2007

12. Greene KE, Wright JR, Steinberg KP, Ruzinski JT, Caldwell E, Wong WB, Hull W, Whitsett JA, Akino T, Kuroki Y, Nagae H, Hudson LD, Martin TR : Serial changes in surfactant-associated proteins in lung and serum before and after onset of ARDS. Am J Respir Crit Care Med $160: 1843-1850,1999$

13. Günther A, Siebert C, Schmidt R, Ziegler S, Grimminger F, Yabut M, Temmesfeld B, Walmrath D, Morr H, Seeger W : Surfactant alterations in severe pneumonia, acute respiratory distress syndrome, and cardiogenic lung edema. Am J Respir Crit Care Med 153 : 176184, 1996

14. Goto H, Ledford JG, Mukherjee S, Noble PW, Williams KL, Wright JR: The role of surfactant protein $\mathrm{A}$ in bleomycin-induced acute lung injury. Am J Respir Crit Care Med 181 : 133644, 2010

15. Jemal A, Siegel R, Ward E, Hao Y, Xu J, Thun $\mathrm{MJ}$ : Cancer statistics, 2009. CA Cancer J Clin $59:$ 225-249, 2009

16. Bejarano PA, Baughman RP, Biddinger PW, Miller MA, Fenoglio-Preiser C, al-Kafaji B, Di Lauro R, Whitsett JA : Surfactant proteins and thyroid transcription factor-1 in pulmonary and breast carcinomas. Mod Pathol 9 : 445-452, 1996

17. Jiang F, Caraway NP, Nebiyou Bekele B, Zhang HZ, Khanna A, Wang H, Li R, Fernandez RL, Zaidi TM, Johnston DA, Katz RL : Surfactant protein A gene deletion and prognostics for patients with stage I non-small cell lung cancer. Clin Cancer Res 11 : 5417-5424, 2005

18. Tsutsumida H, Goto M, Kitajima S, Kubota I, Hirotsu Y, Yonezawa S : Combined status of MUC1 mucin and surfactant apoprotein A expression can predict the outcome of patients with small-size lung adenocarcinoma. Histopathology 44 : 147-155, 2004

19. Mitsuhashi A, Goto H, Kuramoto T, Tabata S, Yukishige S, Abe S, Hanibuchi M, Kakiuchi S, Saijo A, Aono Y, Uehara H, Yano S, Ledford JG, Sone S, Nishioka Y : Surfactant protein A suppresses lung cancer progression by regulating the polarization of tumor-associated macrophages. Am J Pathol 182 : 1843-1853, 2013

20. Pollard JW : Tumour-educated macrophages promote tumour progression and metastasis. Nat Rev Cancer 4 : 71-78, 2004

21. Sano H, Sohma H, Muta T, Nomura S, Voelker DR, Kuroki Y : Pulmonary surfactant protein A modulates the cellular response to smooth and rough lipopolysaccharides by interaction with CD14. J Immunol 163 : 387-395, 1999

22. Sato M, Sano H, Iwaki D, Kudo K, Konishi M, Takahashi H, Takahashi T, Imaizumi H, Asai Y, Kuroki Y : Direct binding of Toll-like receptor 2 to zymosan, and zymosan-induced NF-kappa $B$ activation and TNF-alpha secretion are downregulated by lung collectin surfactant protein A. J Immunol 171 : 417-425, 2003

23. Henning LN, Azad AK, Parsa KV, Crowther JE, Tridandapani S, Schlesinger LS : Pulmonary surfactant protein A regulates TLR expression and activity in human macrophages. J Immunol 180 : 7847-7858, 2008

24. White RT, Damm D, Miller J, Spratt K, Schilling J, Hawgood S, Benson B, Cordell B : Isolation 
and characterization of the human pulmonary surfactant apoprotein gene. Nature 317 : 361363, 1985

25. McCormick SM, Mendelson CR : Human SP$\mathrm{A} 1$ and SP-A2 genes are differentially regulated during development and by cAMP and glucocorticoids. Am J Physiol 266 : 367-374, 1994

26. Oberley RE, Snyder JM : Recombinant human SP-A1 and SP-A2 proteins have different carbohydrate-binding characteristics. Am J Physiol Lung Cell Mol Physiol 284 : 871-881, 2003

27. Wang G, Phelps DS, Umstead TM, Floros J : Human SP-A protein variants derived from one or both genes stimulate TNF-alpha production in the THP-1 cell line. Am J Physiol Lung Cell Mol Physiol 278 : 946-954, 2000
28. Wang G, Umstead TM, Phelps DS, AlMondhiry H, Floros J : The effect of ozone exposure on the ability of human surfactant protein A variants to stimulate cytokine production. Environ Health Perspect 110 : 79-84, 2002

29. Selman M, Lin HM, Montaño M, Jenkins AL, Estrada A, Lin Z, Wang G, DiAngelo SL, Guo X, Umstead TM, Lang CM, Pardo A, Phelps DS, Floros J : Surfactant protein A and B genetic variants predispose to idiopathic pulmonary fibrosis. Hum Genet 113 : 542-550, 2003

30. Maitra M, Cano CA, Garcia CK : Mutant surfactant A2 proteins associated with familial pulmonary fibrosis and lung cancer induce TGF$\beta 1$ secretion. Proc Natl Acad Sci U S A 109 : 21064-21069, 2012 